\section{Hydroview lens implant calcification: 186 exchanges at a district general hospital}

\begin{abstract}
Aim This paper describes the experience at a district general hospital of coping with an abrupt onset of calcification of Hydroview intraocular lens (IOL) implants requiring exchange surgery mostly for symptoms of glare, even though the visual acuities were relatively good.
\end{abstract}

Methods In this retrospective study, the operative details of 174 consecutive lens exchanges by one surgeon were retrieved from the surgeon's notes. Of these, pre and postoperative details of 106 consecutive patients were obtained from the hospital notes.

Results Of the 174 lens exchanges, all were sutureless except one and 31 eyes (18\%) had had previous capsulotomies. Of 143 eyes with intact posterior capsules, eight $(5.6 \%)$ needed anterior vitrectomy. Lens replacements were in the bag in $136(95 \%)$, in the sulcus in five $(3.5 \%)$, and in the anterior chamber in two $(1.5 \%)$. Of the 31 eyes with previous capsulotomies, $10(32 \%)$ needed anterior vitrectomy. Lens replacements were in the bag in $22 \mathbf{( 7 1 \% )}$ and in the sulcus in the remaining nine cases (29\%). Postoperatively the bestcorrected visual acuity was improved in $53 \%$, remained the same in $35 \%$, and deteriorated in $12 \%$.

Conclusion The lens exchange procedure was mostly predictable with satisfactory visual results allowing preoperative counselling of risks to be similar to that for cataract surgery. The onset and resolution of the period of implantation of lenses requiring exchange has not been explained.

Eye (2008) 22, 325-331; doi:10.1038/sj.eye.6702530; published online 20 October 2006

Keywords: Hydroview; lens; implant; exchange; calcification
P Syam¹, P Byrne', G Lewis², T Husain ${ }^{1}$,

G Kleinmann ${ }^{3}$, N Mamalis ${ }^{3}$, DJ Apple ${ }^{3}$ and

T Rimmer ${ }^{1}$

\section{Introduction}

The common reasons for explantation of an intraocular lens (IOL) are decentration or dislocation of the lens, incorrect biometry, or lens opacification. ${ }^{1}$ There are three currently available materials for foldable lens implants (silicone, hydrophobic acrylic, and hydrogel or hydrophilic acrylic), all of which have been reported to opacify. ${ }^{2-7}$ The Hydroview IOL (Bausch and Lomb Surgical, Rochester, NY, USA) is a hydrogel lens and consists of a hydrophilic optic made from a copolymer composed of 2-hydroxeyethyl-methacrylate and 6-hydroxyhexylmethacrylate, ${ }^{8}$ with $18 \%$ water content.

There have been a few reports of late surface opacification of the Hydroview lens, ${ }^{8-13}$ which appeared to be associated with chondroitin sulphate viscoelastic (Viscoat, Alcon

Laboratories). The Hydroview lens implant had been marketed between December 1997 and April 2001 in its Surefold packaging, ${ }^{9,11}$ which allowed for instantaneous and reliable folding of the implant before insertion. These implants were used in Peterborough between June 1998 and May 2002. The viscoelastic used in these procedures was first Healon (Pharmacia, Sweden) before changing to Provisc (Alcon, USA; both sodium hyaluronidate) in August 2000. In May 2001, Bausch and Lomb (B\&L) announced that the cause of the very low rate of lens calcification requiring replacement had been attributed to the silicone on the gasket of the packaging and that this had been replaced.

After 4 years without any problems at Peterborough the first calcified Hydroview implants requiring exchange began to appear in late 2002. By early 2003, the prevalence of calcified lenses was clearly on the increase and way above the reported rate of one in 4000 (Bausch and Lomb News, November 7, 2001).
${ }^{1}$ Eye Department, Peterborough District Hospital, Peterborough, Cambs, UK

Eye Department, Addenbrookes Hospital, Cambridge, UK

${ }^{3}$ Laboratories for Ophthalmic Biodevices Research, Moran Eye Center, University of Utah Health Sciences Center, Salt Lake City, UT, USA

Correspondence: TJ Rimmer, Eye Department,

Peterborough District Hospital, Thorpe Road, Peterborough, Cambs PE3 6DA, UK Tel: +44 1733874018 ; Fax: + 441733874525 E-mail: timothy.rimmer@ pbh-tr.nhs.uk

Received: 7 February 2006 Accepted: 14 June 2006 Published online: 20 October 2006

Declaration: This paper was presented in part at the ASCRS, Washington, April 2005 by TJR and the expenses were covered by Bausch \& Lomb 
This paper reviews the impact of an outbreak of Hydroview lens calcification on a district general hospital. It covers the initial audit, communications with outside agencies, recall arrangements, surgical management, and complications of 174 consecutive exchanges by one surgeon, outcome data of 106 consecutive patients, and issues of hospital reimbursement and patient compensation.

\section{Materials and methods}

\section{Data acquisition}

The months of original implantation of lenses were taken from the hospital's computer system and operative details of 174 consecutive lens exchanges by one surgeon were taken from the surgeon's records. To reduce demands on hard pressed staff, case note retrieval was limited to 106 consecutive patients, providing pre- and post-operative details of 115 exchanged lenses.

\section{Presentation of patients}

Patients presented with symptoms of glare or misty vision, often with good visual acuities, and with a presumed diagnosis of posterior capsular opacification. One or both surfaces of the optic were covered with white or reddish granular deposits. An example is shown in the colour plate, Figure 1a. The anterior surface was usually traversed by two tram lines that were later attributed to where the folding forceps had held the lens. An audit in April 2003 of calcified lenses requiring exchange revealed that all had been implanted over a 7-month period between October 2000 and April 2001, from then on regarded as the risk period (Figure 2a). The problem was declared a Serious Untoward Incident (SUI) by senior hospital managers that triggered a series of meetings involving managers, an ophthalmic surgeon, nurses, and others representing the risk assessment, legal, financial, and public relations interests of the hospital.

\section{Outside agencies}

The situation was explained to the manufacturer and the Medicines and Healthcare Products Regulatory Agency (MHRA). It was pointed out to both that the frequency of lens exchanges was of a different order of magnitude to the known rate attributed to the Surefold packaging. The manufacturer and the MHRA stated that no further action needed to be taken unless problems were seen with the new silicone-free packaging (serial numbers 6G onwards) which gradually replaced the older packaging from May 2001. Contact was then made with the Royal
College of Ophthalmologists that lent support by monitoring the situation through its Professional Standards Committee. All eye departments in the United Kingdom were alerted through the College's database about the apparent risk period. Representatives of the hospitals affected met at the College to share experiences and agree, as far as possible, a unified approach. The risk period coincided with that at other hospitals but this, and the geographical variation, will be the subject of a further publication.

\section{Patient recall}

It was decided at the SUI meetings to contact all surviving 950 of the 1100 patients who had received Hydroview lens implants over the risk period by letter. Patients were informed of the low risk of the problem and were invited to telephone a helpline if they had problems of mistiness or blurring of vision. It was not considered necessary to examine all these patients firstly because exchange surgery would only be considered where symptoms outweighed surgical risks and secondly because any calcification would not be causing any harm to the eye. The local press was not contacted to reduce the chance of causing alarm. The helpline was telephoned by 200 patients. Reassurance was given to 41 and arrangements were made to examine the other 159. Of these patients, 33 (21\%) were listed for exchange, $15(9 \%)$ had lens calcification not requiring exchange, $53(33 \%)$ were listed for laser capsulotomy, and $58(37 \%)$ had clear implants and posterior capsules.

\section{Surgery}

The earliest lens exchanges were challenging. The capsular bag was frequently inseparable from the implant and came out with it. The paper by Habib et $a l^{11}$ helpfully suggested starting the separation of the anterior capsule from the optic with the tip of a $25 \mathrm{G}$ needle. This worked well and one surgeon (TR) offered to undertake further exchanges. This was agreed because a surgical method was likely to evolve more quickly in the hands of one surgeon than if the experience was being diluted between six surgeons.

\section{Surgical procedure of lens exchange}

After sub-Tenon's anaesthesia and irrigation with Povidone iodine 5\%, three paracenteses were made with a diamond $1 \mathrm{~mm}$ blade in both superior quadrants and the inferotemporal quadrant (Figure 1bi). A partial thickness scratch incision was fashioned superiorly and after partial filling of the anterior chamber (AC) with viscoelastic, a normal $2.65 \mathrm{~mm}$ clear cornea incision was 

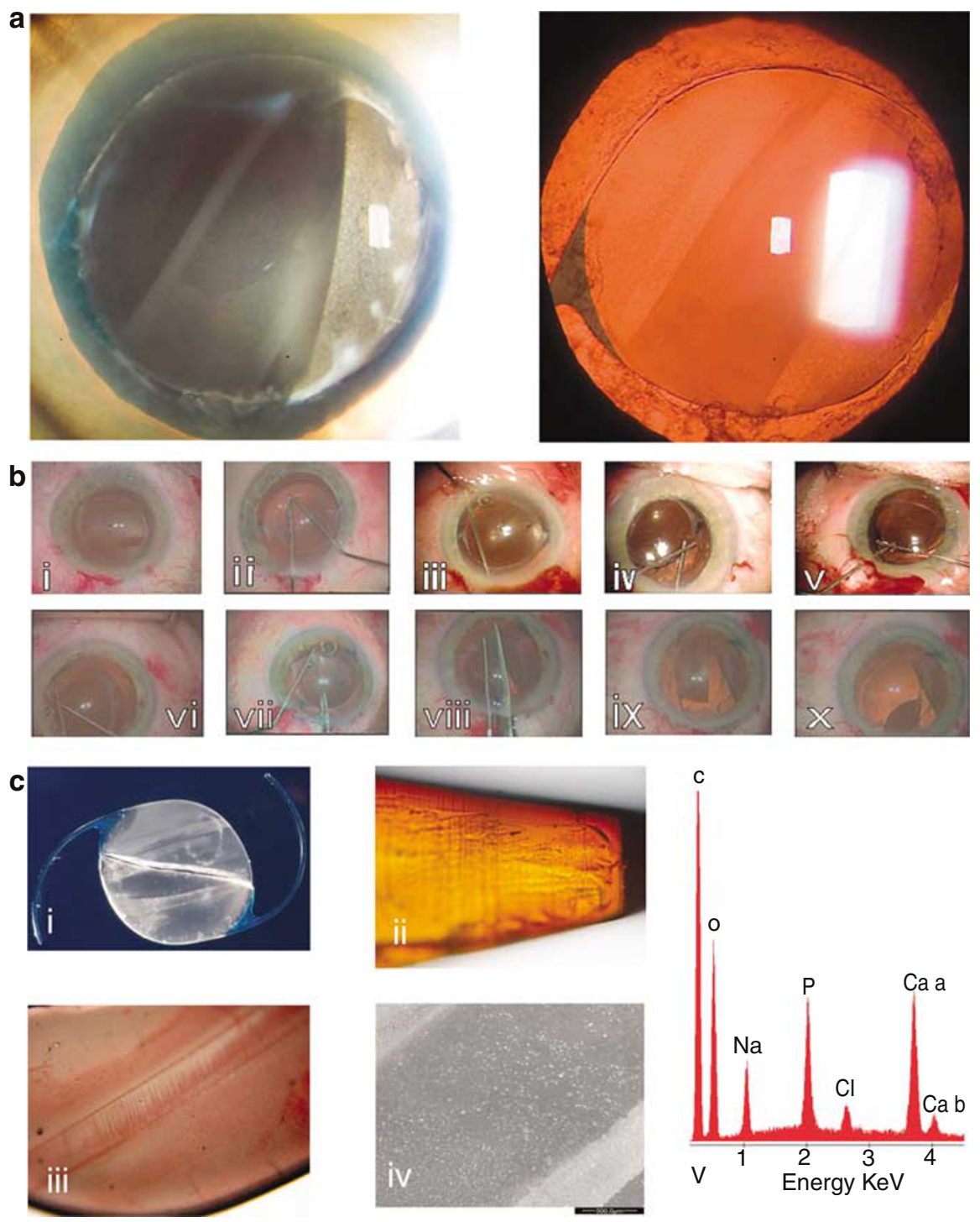

Figure 1 Colour plate: (a) direct (left) and retro (right) illuminated images of a representative calcified Hydroview lens implant. Note the granular deposits showing a pattern of 'tram lines', presumed to mark the contact between the forceps and the folded lens. (b) Surgical snapshots illustrating the steps in removal of a calcified lens implant, see the text for details. (c) Laboratory images showing (i) macroscopic appearance of an explanted bisected implant, (ii) von Kossa and (iii) alizarin red stains for calcium, (iv) scanning electron microscopy with the scale bar representing $500 \mu \mathrm{m}$, and (v) energy dispersion X-ray spectroscopy revealing calcium and phosphorus peaks consistent with hydroxyapatite.

made and immediately widened by $40 \%$. Separation of the capsule was started with a bimanual technique. A short $(12 \mathrm{~mm}) 25 \mathrm{G}$ needle on a $1 \mathrm{ml}$ syringe was held in the left hand, bevel up, and presented through the section with the tip introduced under the inferior edge of the anterior capsule. The tip of the Rycroft needle, on the viscoelastic syringe held by the right hand, followed and allowed injection of viscoelastic under the anterior capsule (Figure 1bii). The $25 \mathrm{G}$ needle was not simply attached to the viscoelastic syringe because the length of the bevel would have caused the tip to puncture the posterior capsule. Viscoelastic was injected under all segments of the anterior capsule (Figure 1biii), access being facilitated by the three paracenteses.

A Hirschman hook was used to draw the optic of the implant inferiorly and to the surgeon's left allowing a cushion of viscoelastic to be injected behind the optic (Figure 1biv). Extensive filling of the capsular bag with viscoelastic probably conferred some rigidity on it and reduced the chance of any adhesions at the haptics from tearing the equator of the capsular bag, or disinserting it, when the implant was manipulated into the AC with two 

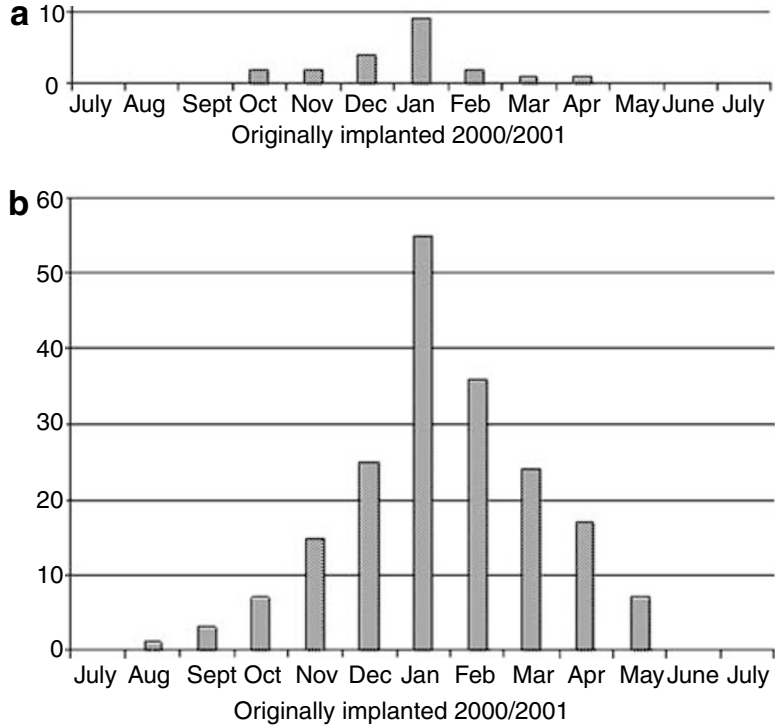

Figure 2 Lenses needing exchange at Peterborough by month of original implantation. About 160 lenses were implanted each month over the period: (a) April 2003 and (b) November 2005.

Hirschman hooks. All manipulations with the Hirschman hooks were through the two superior paracenteses so that, especially in a bulgy eye, the AC was maintained.

The edge of the optic, usually to the surgeon's left, was lifted forward with a Hirschman hook in the surgeon's right hand. This also lifted the adjacent haptic forward into the AC (Figure 1bv). The second Hirschman hook in the surgeon's left hand was used to retract the adjacent pupil margin and capsule if necessary to prevent the capsule from being dragged anteriorly and centrally. The second haptic was manipulated into the AC by the same process (Figure 1bvi). The implant was dialled in order to leave the shoulder of one of the haptics close to the section and just to the surgeon's left of midline. Vannas scissors were introduced through the section and bisection of the optic was commenced just under the shoulder. Movement of the optic away from the scissors was prevented by a Hirschman hook or chopper engaged on the opposite edge (Figure 1bvii and viii). The two halves of the implant were then removed (Figure 1bix and $\mathrm{x}$ ).

The new lens was implanted in the normal way in the bag or in the sulcus if there was insufficient posterior capsule. AC lenses were used in two cases where there was inadequate capsular support. Anterior vitrectomy was performed when necessary. Betnesol and cefuroxime or gentamycin were injected subconjunctivally and maxidex/neomycin ointment was applied.

If the AC was difficult to maintain despite copious viscoelastic, often in the presence of a posterior capsulotomy, the situation was greatly improved by a pars plana tap with a $21 \mathrm{G}$ needle. In five cases, the rhexis was enlarged with radial cuts with Vannas scissors. Cuts to the superior edge were achieved by pulling it over the lower blade of the scissors with a Hirschman hook (for demonstrating steps in the lens exchange procedure see Supplementary Information).

\section{Financial issues}

The manufacturer fully reimbursed the hospital for all costs involved of replacing the lenses and all the associated arrangements for recall of patients. After the daughter of one patient asked about the compensation for patients, it was decided to inform all previous and subsequent patients 2 weeks after surgery that under consumer protection law negligence of the manufacturer does not have to be proven to claim compensation.

Patients were informed of this after surgery because it was felt that the patient's decision to have surgery must be based solely on clinical grounds and that this might be complicated by thoughts of compensation. Most of the patients have contacted a local solicitor who, with several others, has reached agreement in principle with the manufacturer relatively quickly.

\section{Results}

At the time of submission, 186 Hydroview lenses had been explanted for late surface opacification (15.8\% of 1100 implanted between September 2000 and May 2001) at Peterborough District Hospital. Figure $2 \mathrm{~b}$ shows the month of original implantation of all lenses exchanged up to November 2005. This shows a Gaussian distribution with the peak month of lens failure being January 2001 when 160 Hydroview lenses were implanted. Figure 3 shows the intervals between implantation and explantation of 55 of them up to the

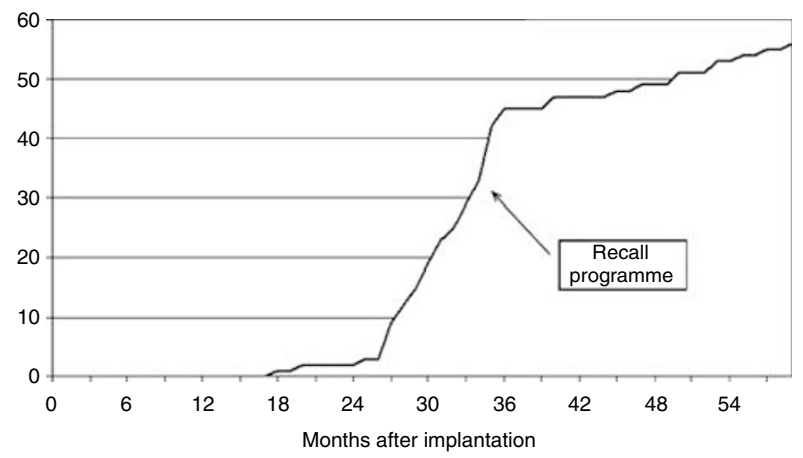

Figure 3 Incremental time plot of Hydroview lenses requiring exchange of 160 originally implanted in January 2001. 
Table 1 Age distribution of a cohort of 106 patients who underwent lens exchange

\begin{tabular}{lc}
\hline Age of the patients (years) & Number of patients \\
\hline $51-60$ & 8 \\
$61-70$ & 9 \\
$71-80$ & 36 \\
$81-90$ & 45 \\
$91-100$ & 8 \\
\hline
\end{tabular}

time of submission of this paper (November 2005). The cumulative plot appears to be still climbing.

The ages of the 106 patients studied in detail varied from 52 to 98 years (mean 72 years) and are shown in Table 1. Of these, 63 patients were female and 43 were male. Optometrists had referred $35(33 \%)$, general practitioners had referred $28(26 \%), 25$ had been identified through the recall programme (23\%), and 18 had been listed from hospital clinics (17\%). Nine required bilateral procedures and nine patients were deceased.

None of these patients were known to suffer from any calcium metabolic disease. Examining their comorbidity, $18(17 \%)$ suffered from diabetes. Of these, $5.4 \%$ suffered from type I diabetes and $10.8 \%$ from type II diabetes. Hypertension was the most common general comorbidity (21 cases, 20\%) followed by heart disease in 19 cases (18\%). With regard to ocular comorbidity, nine patients had diabetic retinopathy, seven patients glaucoma, and six cases age-related macular degeneration.

Preoperative visual acuities varied from $6 / 5$ to hand movement. Of these 115 eyes, the visual acuities were $6 / 5-6 / 12$ in $87(76 \%), 6 / 18-6 / 60$ in $22(19 \%)$, and worse than $6 / 60$ in six $(5 \%)$. The average drop in acuity at representation compared with the original postoperative assessment was 1.79 Snellen lines. The overall range of reduction in visual acuity was 2-8 Snellen lines. There was no correlation between the length of time the lens was in vivo and the amount of reduced acuity. The lens causing a drop of 8 Snellen lines had been in situ for only 117 weeks before representation.

Operative details of the consecutive 174 lens exchanges by one surgeon are shown in Table 2 . All were sutureless except one. There had been previous capsulotomies in 31 . Most of these were by laser but three had occurred during the original implantation, two requiring anterior vitrectomy. One of the replaced lenses dislocated posteriorly and was removed and replaced with an AC lens by a vitreoretinal specialist. Two further lenses required repositioning into the sulcus. Twenty patients had laser posterior capsulotomies after their exchanges.

Postoperative visual acuity of the 115 eyes of 106 patients analysed, improved in $61(53 \%)$ eyes (range 1-7 Snellen lines, mean 2.1), remained the same in 40 (35\%)
Table 2 Operative details of 174 consecutive lens exchanges by one surgeon

\begin{tabular}{lcc}
\hline & $\begin{array}{c}\text { Intact posterior } \\
\text { capsules } \\
\text { Consecutive lens } \\
\text { exchanges }\end{array}$ & $\begin{array}{c}\text { Previous } \\
\text { capsulotomies } \\
31(18 \%)\end{array}$ \\
\hline Anterior vitrectomy & $8(62 \%)$ & $10(32 \%)$ \\
New implant position & $136(95 \%)$ & $22(71 \%)$ \\
$\quad$ Bag & $5(3.5 \%)$ & $9(29 \%)$ \\
Sulcus & $2(1.5 \%)$ & 0 \\
AC & & \\
\hline
\end{tabular}

and deteriorated in 14 eyes (12\%). On later investigation of the 14 cases with worse visual acuities, nine patients were deceased. One of these cases had been followed by endophthalmitis, causing total loss of vision. Of the surviving five patients, two developed surgically induced astigmatism ( 2 and $4 \mathrm{~d}$ cylinder), one patient developed corneal decompensation and was considering corneal transplantation, and two patients developed chronic cystoid macular oedema. Of the 101 cases of this cohort with intact posterior capsules, postoperative visual acuities were better in 53\%, unchanged in 34\%, and worse in $13 \%$. Of the 14 cases with previous capsulotomies, $50 \%$ of postoperative visual acuities were better, $43 \%$ were unchanged, and $7 \%$ were worse.

The onset of implantation of faulty lenses bears no relation to either the introduction of the Surefold folding device with the suspect silicone gasket (December 1997) or the commencement of use of the lens at Peterborough Hospital (June 1998). It should also be noted that implantation of faulty lenses had stopped just before the silicone gasket was gradually replaced (May 2001). The examination of the surgical log book revealed that only 13 lenses implanted in May 2001 had been in the new packaging (serial numbers 6G onwards) and that these appeared gradually rather than suddenly indicating that the lenses in the old packaging had not been cleared out at the time. This was logical because the explantation rate at the time was believed to be only one in 4000 .

For laboratory analysis, 121 explanted lenses were sent to the David J Apple, MD, Laboratories for Ophthalmic Devices Research, Salt Lake City, UT, USA.

Representative images are shown in Figure 1c. Lenses were stained with von Kossa (Figure 1cii) and alizarin red (Figure 1ciii), which showed calcium on the outer surface of the implant but not the section surface. Scanning electron microscopy revealed granular deposits on the surface composed of multiple spherical-ovoid globules, scattered in some areas, and confluent in others (Figure 1civ). Energy dispersion X-ray spectroscopy 
revealed peaks of calcium and phosphorous consistent with calcium hydroxyapatite (Figure 1cv).

\section{Discussion}

Lens explantation is distressing for patients, exposes them to additional operative risks and creates an increased workload for hospital systems that have no spare capacity. The cause of an outbreak of lens opacification must be explored so that industry learns from the episode in order to reduce the chance of a similar problem occurring again.

This paper reports an outbreak of opacification of the Hydroview lens implant, owing to calcification occurring on the external surfaces, requiring explantation. Visually significant calcification of Hydroview lenses were reported as early as 1999..$^{8,11,12}$ Habib et al ${ }^{11}$ showed the major component of the deposits to be calcium phosphate salts. Company investigations of previous reported cases suggested that migration of silicone particles from the Surefold packaging system onto the external surface of the IOL had an important role in the evolution of these deposits (in-house publication, B\&L, 2001). This was later confirmed by others. ${ }^{7,14} \mathrm{~B} \& \mathrm{~L}$ replaced the packaging system on May 2001. The onset and resolution of the outbreak of calcification reported here does not fit with the introduction and withdrawal of the silicone gasket of the Surefold packaging system. Silicone might indeed be a prerequisite of calcification but the timing of this outbreak remains unexplained.

This risk period, also seen in other hospitals affected in the UK, is consistent with a sudden rise in lenses explanted worldwide, which had been manufactured in the third-quarter of 2000, according to figures supplied by B\&L and shown in Figure 4. The worldwide rate of reporting of lens failures to the manufacturer is unknown. Although these 500 represent a large rise in explanted lenses, the number is only about $1 \%$ of approximately 50000 manufactured during that quarter. The manufacturer has examined its processes but is unable to account for this episode of calcification.

In our case series, the need for explantation was based on the presence of surface deposits and the severity of the patient's symptoms of deterioration of quality of vision. The main symptoms were glare and loss of contrast sensitivity, often in the presence of normal or near-normal visual acuity. The explantation rate at Peterborough reached $34 \%$ for the 160 implanted in January 2001, which is much higher than that initially reported by the manufacturer $(0.025 \%)$. The reason for this is unknown. Previous outbreaks were associated with Viscoat but this viscoelastic was not used at Peterborough or other UK hospitals affected by this outbreak.

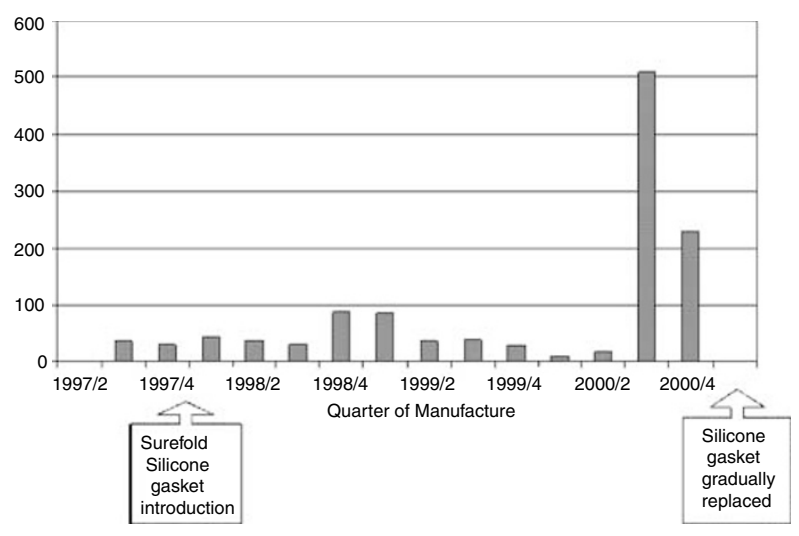

Figure 4 Global explantation rates of Hydroview lenses by quarter of manufactured, as at December 2004. Raw data courtesy of B\&L. Note that the period of use of silicone in the packaging covers the outbreak of calcification but does not coincide with the beginning and end of it.

Visual acuity in our series improved in 61 cases (53\%), remained the same in $40(35 \%)$, and worsened in 14 cases $(12 \%)$. It should be appreciated, however, that loss of contrast rather than visual acuity was the main symptom and that patients were generally very pleased with the result of lens exchange surgery. With hindsight pre- and post-operative contrast sensitivity measurements, as performed by Altaie et al, ${ }_{15}^{15}$ would have reflected much greater improvement of visual function.

The anterior vitrectomy rate was five times higher when there had been a previous capsulotomy but this did not seem to adversely affect the final visual acuity. Of the 174 consecutive cases by one surgeon all replacement lenses but two were in the posterior chamber and all but one were without sutures. The transfer of the cases to one surgeon probably contributed to the relatively low complication rate.

With around 600000 lenses in packaging containing silicone sold worldwide, it is possible that the need for these lenses to be exchanged might continue for years to come. Surprisingly, the onset of symptoms owing to lens implant calcification has been as late as 260 weeks after implantation at our centre. It is encouraging that the visual outcome of late lens exchange appears to be good and that surgical complication rates can be relatively low. However, this global outbreak of calcification of Hydroview lenses, and its geographical variation, needs to be explained so that lessons can be learnt.

\section{References}

1 Mamalis N. Complications of foldable intraocular lenses requiring explantation or secondary intervention - 1998 survey. J Cataract Refract Surg 2000; 26: 766-772.

2 Parkin B, Pitts-Crick M. Opacification of silicone intraocular lens implant requiring lens exchange. Eye 2000; 14: 794-795. 
3 Chang BYP, Davey KG, Gupta M. Late clouding of an acrylic intraocular lens following routine phacoemulsification. Eye 1999; 13: 807-808.

4 Saeed MU, Jafree AJ, de Cock R. Intralenticular opacification of hydrophilic acrylic intraocular lenses. Eye 2005; 19: 661-664.

5 Dhaliwal DK, Mammalis N, Olson RJ, Crandall AS, Zimmerman P, Alldredge OC et al. Visual significance of glistenings seen in the acrysof intraocular lens. J Cataract Refract Surg 1996; 22: 452-457.

6 Goodall KL, Ghosh YK. Total opacification of intraocular lens implant after uncomplicated cataract surgery: a case series. Arch Ophthalmol 2004; 122: 782-784.

7 Guan X, Tang R, Nancollas GH. The potential calcification of octacalcium phosphate on intraocular lens surfaces. J Biomed Mater Res 2004; 71(3): 488-496.

8 Yu AKF, Shek TWH. Hydroxyapatite formation on implanted hydrogel intraocular lenses. Arch Ophthalmol 2001; 119: 611-614.

9 Izak AM, Werner L, Pandey SK, Mackey TA, Trivedi RH, Apple DJ. Calcification on the surface of the Bausch and Lomb Hydroview ${ }^{\mathbb{R}}$ intraocular lens. Int Ophthalmol Clin 2001; 41: 63-77.
10 Fernando GT, Crayford BB. Visually significant calcification of hydrogel intraocular lenses necessitating explantation. Clin Exp Ophthalmol 2000; 28(4): 280-286.

11 Habib NE, Freegard TJ, Gock G, Newman PL, Moate RM. Late surface opacification of Hydroview ${ }^{\mathbb{R}}$ intraocular lenses. Eye 2002; 16: 69-74.

12 Werner L, Apple DJ, Escobar-Gomez M, Ohrstrom A, Crayford BB, Bianchi $\mathrm{R}$ et al. Postoperative deposition of calcium on the surfaces of a hydrogel intraocular lens. Ophthalmology 2000; 107: 2179-2185.

13 Murray RI. Two cases of late opacification of the Hydroview $^{\mathbb{R}}$ intraocular lens. J Cataract Refract Surg 2000; 26: 1272-1273.

14 Dorey MW, Brownstein S, Hill VE, Mathew B, Botton G, Kertes PJ et al. Proposed pathogenesis for the delayed postoperative opacification of the Hydroview ${ }^{\mathbb{R}}$ hydrogel intraocular lens. Am J Ophthalmol 2003; 135: 591-598.

15 Altaie RW, Costigan T, Donegan S, O'brien P, Mahmood U, Bogdan AJ et al. Investigation and management of an epidemic of Hydroview intraocular lens opacification. Graefes Arch Clin Exp Ophthalmol 2005; 243: 1124-1133.

Supplementary Information accompanies the paper on the Eye website (http://www.nature.com/eye). 